\title{
Quasi-free Compton Scattering from the Deuteron and Nucleon Polarizabilities
}

\author{
N.R. Kolb, A.W. Rauf, R. Igarashi, D.L. Hornidge, R.E. Pywell, B.J. Warkentin \\ Saskatchewan Accelerator Laboratory, University of Saskatchewan, Saskatoon, SK, Canada, S7N 5C6 \\ E. Korkmaz \\ Department of Physics, University of Northern British Columbia, Prince George, BC, Canada, V2N 4Z9 \\ G. Feldman, G.V. O'Rielly \\ Department of Physics, The George Washington University, Washington, D.C. 20052
}

(February 5, 2020)

\begin{abstract}
Cross sections for quasi-free Compton scattering from the deuteron were measured for incident energies of $\mathrm{E}_{\gamma}=236-260 \mathrm{MeV}$ at the laboratory angle $\theta_{\gamma^{\prime}}=-135^{\circ}$. The recoil nucleons were detected in a liquid-scintillator array situated at $\theta_{N}=20^{\circ}$. The measured differential cross sections were used, with the calculations of Levchuk et al., to determine the polarizabilities of the bound nucleons. For the bound proton, the extracted values were consistent with the accepted value for the free proton. Combining our results for the bound neutron with those from Rose et al., we obtain one-sigma constraints of $\bar{\alpha}_{n}=7.6-14.0$ and $\bar{\beta}_{n}=1.2-7.6$.

PACS numbers: 25.20.Dc, 13.40.Em, 13.60.Fz, 14.20.Dh
\end{abstract}

The electric $\bar{\alpha}$ and magnetic $\bar{\beta}$ polarizabilities constitute the first-order responses of the internal structure of a nucleon to externally applied electric and magnetic fields. Compton scattering from the proton has been used extensively to determine the polarizabilities of the proton (see Ref. [1] and references contained therein). Such measurements are sensitive to the sum (difference) of the polarizabilities for photons scattered at forward (backward) angles. Ref. [1] reports the experimental status of the proton polarizabilities, in the usual units of $10^{-4} \mathrm{fm}^{3}$ :

$$
\begin{aligned}
& (\bar{\alpha}-\bar{\beta})_{p}=10.0 \pm 1.5 \pm 0.9, \\
& (\bar{\alpha}+\bar{\beta})_{p}=15.2 \pm 2.6 \pm 0.2,
\end{aligned}
$$

where the first error is the combined statistical and systematic, and the second is due to the model dependence of the dispersion-relation extraction method.

Disperson sum rules relate the sum of the polarizabilities to the nucleon photoabsorption cross section. The generally accepted results [2] were

$$
\begin{aligned}
& (\bar{\alpha}+\bar{\beta})_{p}=14.2 \pm 0.5 \\
& (\bar{\alpha}+\bar{\beta})_{n}=15.8 \pm 0.5 .
\end{aligned}
$$

There have been recent reevaluations of these sum rules which yield $13.69 \pm 0.14(14.40 \pm 0.66)[3]$ and $14.0 \pm 0.5$ $(15.2 \pm 0.5)$ 细 for the proton (neutron). The proton polarizabilities, obtained from Eqs. 1 and 3, are

$$
\bar{\alpha}_{p}=12.1 \pm 0.8 \pm 0.5, \quad \bar{\beta}_{p}=2.1 \mp 0.8 \mp 0.5 .
$$

The status of the neutron polarizabilities is much less satisfactory (see Ref. [5] and references contained therein). Measurements of the electric polarizability of the neutron have been done by low-energy neutron scattering from the Coulomb field of a heavy nucleus. The extracted values fall in the range $\alpha_{n}=0-19$ [6] 8$]$.
Elastic Compton scattering from the deuteron has also been used to extract information on the nucleon polarizabilities. Using their theoretical model, Levchuk and L'vov [4] have reported values of

$$
(\bar{\alpha}-\bar{\beta})_{n}=-2 \pm 3, \quad(\bar{\alpha}+\bar{\beta})_{n}=20 \pm 3,
$$

from fitting to the data of Refs. [9, 10]. The large discrepancy between $(\bar{\alpha}-\bar{\beta})_{n}$ and $(\bar{\alpha}-\beta)_{p}$ (Eq. 11) may be indicative of shortcomings in the theoretical models used to extract the polarizabilities from the $d(\gamma, \gamma) d$ reaction.

The quasi-free Compton scattering reaction $d\left(\gamma, \gamma^{\prime} n\right) p$, in which the scattered photon is detected in coincidence with the recoil neutron, can be used to minimize the model dependence of the extracted polarizabilities. There has been one measurement reported on this reaction using bremsstrahlung photons with an endpoint of $130 \mathrm{MeV}$ [11]. A most-probable value of $\bar{\alpha}_{n}=10.7$ was obtained with an upper limit of 14.0 but with no constraint on the lower limit. In addition, the model dependence is strong at these lower energies.

Levchuk et al. 12] have determined that, within the context of their model, the sensitivity to the neutron amplitude is maximized (and model dependence minimized) for $\mathrm{E}_{\gamma}=200-300 \mathrm{MeV}$ and backward angles for the scattered photons. For $E_{\gamma}=247 \mathrm{MeV}$ and $\theta_{\gamma^{\prime}}=-135^{\circ}$, the contribution of the spectator nucleon modifies the cross section by only $\sim 4 \%$ at the quasi-free peak. Furthermore, the free-neutron cross section can be related to the cross section in the center of the neutron quasi-free peak via a spectator formula 12.13

$$
\frac{d \sigma\left(\gamma n \rightarrow \gamma^{\prime} n\right)}{d \Omega_{\gamma^{\prime}}}=\frac{(2 \pi)^{3}}{u^{2}(0)} \frac{E_{\gamma} E_{\gamma^{\prime}}}{\left|\mathbf{p}_{n}\right| m E_{\gamma^{\prime}}^{(n) 2}} \frac{d^{3} \sigma\left(\gamma d \rightarrow \gamma^{\prime} n p\right)}{d \Omega_{\gamma^{\prime}} d \Omega_{n} d E_{n}},
$$


where $u(0)$ is the $\mathrm{S}$-wave amplitude of the deuteron wave function at zero momentum, and $E_{\gamma^{\prime}}^{(n)}$ is the photon energy in the rest frame of the final neutron. The cross section on the right side of the equation must be corrected for the small contribution of the spectator proton (its pole diagram and final state interactions).

The present measurement was performed at the Saskatchewan Accelerator Laboratory (SAL). The $d\left(\gamma, \gamma^{\prime} n\right) p$ and $d\left(\gamma, \gamma^{\prime} p\right) n$ cross sections were measured simultaneously, in kinematics that emphasized the quasi-free reaction. Data were also obtained from the free proton, using the same apparatus, for calibration and normalization purposes. An electron beam of $292 \mathrm{MeV}$ and $\sim 60 \%$ duty factor produced bremsstrahlung photons, which were tagged in the energy range $236-260 \mathrm{MeV}$, with a resolution of $0.4 \mathrm{MeV}$. The average, integrated tagged flux was $\sim 6 \times 10^{6}$ photons/s with a tagging efficiency of $\sim 45 \%$. The total integrated luminosity for the deuterium measurements was $1.25 \times 10^{37}$ photons $/ \mathrm{cm}^{2}$.

The cryogenic target cell was a vertical cylinder $(12 \mathrm{~cm}$ in diameter), with Mylar walls, containing liquid hydrogen $(p(\gamma, \gamma))$ or liquid deuterium $\left(d\left(\gamma, \gamma^{\prime} N\right)\right.$ and $d(\gamma, p n))$. The quasi-free scattering of $247 \mathrm{MeV}$ photons to $-135^{\circ}$ from deuterium results in the recoil nucleon being confined to a forward cone centered around $18^{\circ}$. These nucleons have a central kinetic energy of $\sim 77 \mathrm{MeV}$ and were detected in a liquid-scintillator array [14] situated at $20^{\circ}$, subtending a solid angle of approximately $0.11 \mathrm{sr}$. The array consisted of 85 Lucite-walled cells, filled with BC-505 liquid scintillator. Between the cells and the target were thin plastic scintillators which acted as veto detectors for neutrons or as $\Delta \mathrm{E}$ detectors for protons.

The neutron efficiency of the array was measured at the beginning of the experiment via the $d(\gamma, p n)$ reaction. A plastic-scintillator $\Delta \mathrm{E} \cdot \mathrm{E}$ telescope was placed at $80^{\circ}$ to detect the protons while the neutron array was located at $-77^{\circ}$. The detection efficiency was measured for neutrons with kinetic energies of $50-100 \mathrm{MeV}$, covering the range of interest for the quasi-free reaction. With the applied threshold of $11 \mathrm{MeV}$ (6 MeVelectron equivalent) the efficiency ranged from $5.2-5.9 \%$.

Photons scattered from the target were detected in the large-volume Boston University NaI (BUNI) gamma-ray spectrometer 15]. BUNI is composed of five opticallyisolated segments of NaI, the core and four surrounding quadrants. Plastic-scintillator detectors were used for rejection of cosmic rays as well as charged particles from the target. BUNI was set at $-135^{\circ}$ and subtended a solid angle of $0.013 \mathrm{sr}$. Zero-degree calibrations of BUNI were done at the beginning and end of the experiment, in order to obtain both the lineshape of BUNI and an energy calibration for the NaI core. The NaI quadrants were calibrated periodically with a radioactive source (Th-C).

Figure 1 1 depicts the BUNI energy spectrum $\left(\mathrm{E}_{\gamma^{\prime}}\right)$, cor- rected for photon absorption effects $(\sim 3 \%)$, from the free proton. Random coincidences as well as contributions from empty-target backgrounds $(36 \%$ for $(\gamma, \gamma)$ and $5 \%$ for $(\gamma, \gamma p)$ ) have been subtracted. The spectra are summed over all tagged photon energies and shifted to the maximum incident energy of $260 \mathrm{MeV}$, with the appropriate kinematic corrections to account for the different scattered photon energy at each incident photon energy.

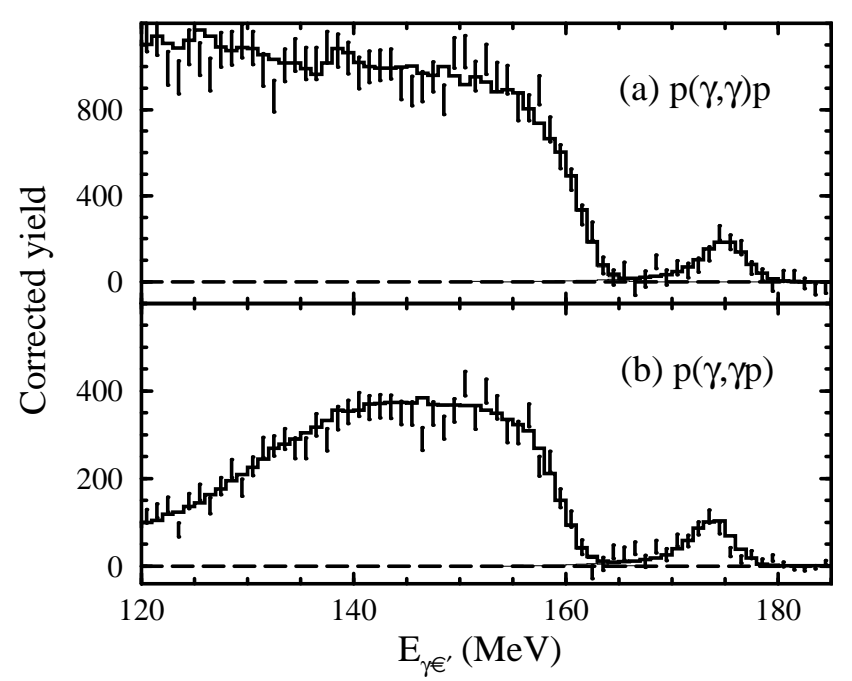

FIG. 1. Detected photon energy spectra for $\mathrm{E}_{\gamma}=247 \mathrm{MeV}$ and $\theta_{\gamma}=-135^{\circ}$ from the free proton without (a) and with (b) detection of the proton. The histograms are from a simulation of the experiment.

Figure 1(a) is the energy spectrum from a free proton, without detection of the recoil proton. The Compton scattered events (the peak near $175 \mathrm{MeV}$ ) are clearly distinguishable from the large number of events from neutral pion $\left(\pi^{0}\right)$ production (below $\sim 165 \mathrm{MeV}$ ). The histogram is the result of a simulation of the experiment. The final yield was determined by integrating the data from 170 to $190 \mathrm{MeV}$ and correcting for the Compton events excluded from this region (18\%) and the $\pi^{0}$ events included in this region (1\%). For $\mathrm{E}_{\gamma}=247 \mathrm{MeV}$ and $\theta_{\gamma}=-135^{\circ}$, the cross section for elastic Compton scattering from the free proton $(p(\gamma, \gamma) p)$ was determined to be

$$
\frac{d \sigma}{d \Omega_{\gamma}}=94.6 \pm 9.0 \pm 3.8 \mathrm{nb} / \mathrm{sr},
$$

where the first error is statistical and the second is systematic. This is in good agreement with recent measurements 13, 16, 17]. The dispersion calculations of L'vov et al. 18] predict a somewhat lower value of $78.0 \mathrm{nb} / \mathrm{sr}$ using $(\bar{\alpha}-\bar{\beta})_{p}=10$.

The efficiency for the detection of protons in the array was determined in situ from the fraction of the 
$p(\gamma, \gamma) p$ events (Fig. 1 $1(\mathrm{a}))$ that included a detected proton (Fig. 1 $1(b))$. This correction $(0.58 \pm 0.02)$ was accounted for by a combination of the simulation and cuts in the analysis.

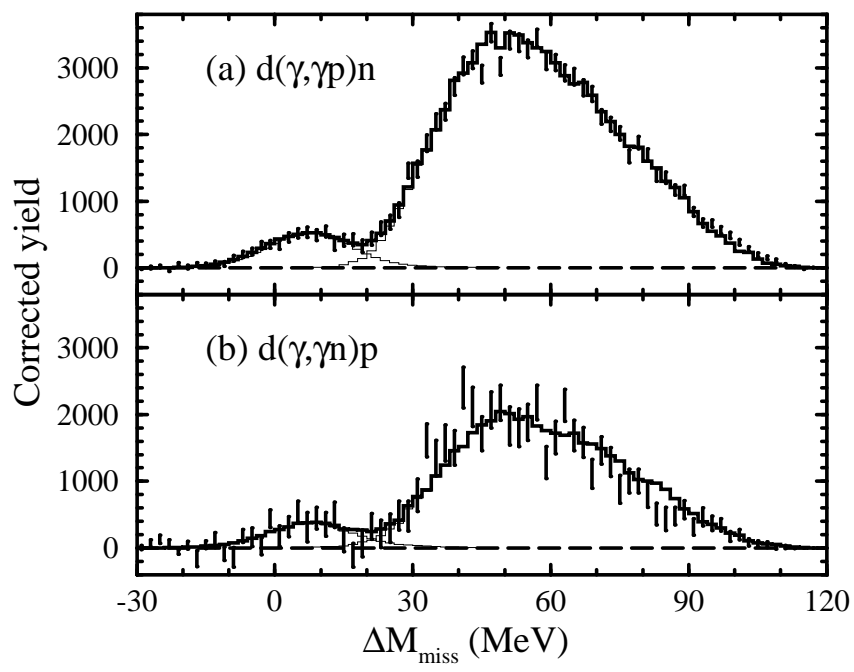

FIG. 2. Missing mass $\left(\Delta \mathrm{M}_{\text {miss }}=\mathrm{MX}_{\mathrm{X}}-\mathrm{M}_{\mathrm{N}}\right)$ spectra for $\mathrm{E}_{\gamma}=247 \mathrm{MeV}$ and $\theta_{\gamma^{\prime}}=-135^{\circ}$ from the bound proton (a) and the bound neutron (b). The histograms are from simulations of the experiment.

For Compton scattering from a bound nucleon, the Fermi motion causes overlap in $\mathrm{E}_{\gamma^{\prime}}$ between the Compton and $\pi^{0}$ events. In order to obtain better separation, the missing mass $\left(\mathrm{M}_{\mathrm{X}}\right)$ of the spectator nucleon was calculated. Figure 2 depicts the difference between the missing mass and the spectator mass $\left(\Delta \mathrm{M}_{\text {miss }}\right)$ for the two quasi-free reactions. The yields are corrected for random coincidences, empty-target backgrounds (12-14\%), and detection efficiencies. The histograms are from simulations of the experiment. The thin lines are the individual contributions from the $\pi^{0}$ and Compton reactions while the thick line is the sum. The simulation assumes that the deuteron reactions proceed via quasi-free kinematics. The spectator nucleon is distributed according to its Fermi distribution and the angular distribution of the $\pi^{0}$ or photon is determined by the theoretical calculations of Levchuk et al. The simulation gives a good fit to the data, and is relatively insensitive to the input angular distribution $(\sim 2 \%)$. The $\chi^{2} / N_{\text {d.o.f. }}$ is 1.4 (proton) and 1.5 (neutron) for $N_{\text {d.o.f. }}=63(-20$ to $110 \mathrm{MeV})$. To extract the yields for the Compton reactions, the data were integrated from -20 to $+20 \mathrm{MeV}$ and corrected for the Compton events excluded from this region (10-15\%) and the $\pi^{0}$ events included in this region $(7-8 \%)$.

In order to compare to theoretical predictions, the measured cross sections were interpolated to the quasifree kinematic point $\left(P_{\text {spectator }}=0\right)$ through use of the simulation. The average and root-mean-square values for $P_{\text {spectator }}$ were 48 and $55 \mathrm{MeV} / \mathrm{c}$, respectively. For $\mathrm{E}_{\gamma}=247 \mathrm{MeV}$ and $\theta_{\gamma^{\prime}}=-135^{\circ}$, the final differential cross sections, at the quasi-free point, are

$$
\begin{aligned}
\frac{d^{3} \sigma}{d \Omega_{\gamma} d \Omega_{N} d E_{N}} & =56.5 \pm 2.8 \pm 4.5 \text { (proton) } \\
& =33.3 \pm 7.2 \pm 3.0 \text { (neutron) }
\end{aligned}
$$

where the units are $\mathrm{nb} / \mathrm{sr}^{2} / \mathrm{MeV}$, the first error is statistical, and the second is systematic. The proton result is consistent with the recent results of Wissmann et al. [13].

Since the back-angle Compton cross section is primarily sensitive to the difference of the polarizabilities, $(\bar{\alpha}-\bar{\beta})_{p}$ was used as a fit parameter in matching the theoretical calculation Levchuk et al. [12] to the bound proton data (Fig. 3(a)). The solid line is the theoretical calculation, the thin line is the central fitted value, and the horizontal dashed lines indicate the combined-error band (the quadratic sum of statistical and systematic). The model dependence of the calculation is small compared to the experimental errors. The fitted value,

$$
(\bar{\alpha}-\bar{\beta})_{p}=14.7_{-4.0}^{+4.6}
$$

is consistent with the free proton result (Eq. 1), as well as with the quasi-free results of Wissmann et al. [13]. This indicates that the theoretical calculations are adequately describing the nuclear effects.

The theoretical calculations have been carried out using a 'traditional' value for the backward-spin polarizability of $\gamma_{\pi}=-36.8$. Using the value $\gamma_{\pi}=-27.1$, suggested by Tonnison et al. [19], results in a fitted value of

$$
(\bar{\alpha}-\bar{\beta})_{p}=26.6_{-5.8}^{+7.8}
$$

which is over two standard deviations from the free value. Our data show no evidence for modification of $\gamma_{\pi}$, in agreement with the conclusions of Wissmann et al. [13.

The ratio of the bound proton to the bound neutron quasi-free cross section is $1.70 \pm 0.38 \pm 0.13$. Using the ratio of the measured cross sections minimizes the effect of systematic errors on the extraction of $(\bar{\alpha}-\bar{\beta})_{n}$, and reduces the model dependence inherent in extracting information on the free-neutron from quasi-free results. The ratio for the theoretical calculation was obtained by fixing the proton polarizabilities (Eq. 1) and treating $(\bar{\alpha}-\bar{\beta})_{n}$ as a fit parameter (Fig. $3(\mathrm{~b})$ ). The best fit was obtained with $(\bar{\alpha}-\bar{\beta})_{n}=12$, with a lower limit of 0 . No upper limit can be obtained. The model uncertainty in extracting $(\bar{\alpha}-\bar{\beta})_{n}$ is expected to be negligible compared to the experimental error. The central value is markedly different from the value extracted from elastic Compton scattering from the deuteron (Eq. 6). However, the two are within errors. Invoking the sum rule [ 4 ], the values for the polarizabilities are $\bar{\alpha}_{n}=13.6$, with a lower limit 
of 7.6, and $\bar{\beta}_{n}=1.6$, with an upper limit of 7.6. The upper (lower) limits on $\bar{\alpha}_{n}\left(\bar{\beta}_{n}\right)$ are unconstrained by the present measurement. Combining our results with those from Rose et al. 11, we obtain one-sigma constraints of $\bar{\alpha}_{n}=7.6-14.0$ and $\bar{\beta}_{n}=1.2-7.6$. These can be compared with recent Chiral Perturbation Theory (ChPT) predictions at $O\left(Q^{4}\right)$ 20]

$$
\bar{\alpha}_{n}=13.4 \pm 1.5, \quad \bar{\beta}_{n}=7.8 \pm 3.6 .
$$
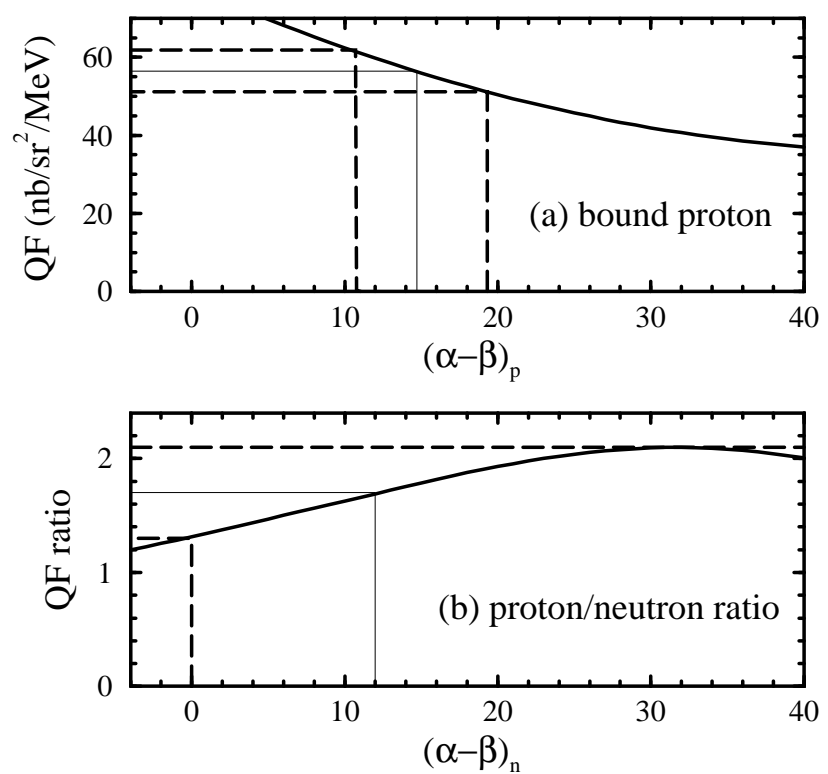

FIG. 3. (a) Quasi-free cross section for the bound proton. (b) Ratio of the quasi-free cross section of the bound proton and neutron. The curves are the theoretical calculation of Levchuk et al.

From Eqs. 7 and 9 the effective free-proton cross section at $\theta_{\gamma^{\prime}}=-135^{\circ}$ can be determined from that of the bound proton $\left(d\left(\gamma, \gamma^{\prime} p\right) n\right)$. The result is

$$
\frac{d \sigma\left(\gamma p \rightarrow \gamma^{\prime} p\right)}{d \Omega_{\gamma}}=66.8 \pm 3.4 \pm 5.3 \mathrm{nb} / \mathrm{sr}
$$

corresponding to $\mathrm{E}_{\gamma}=244.6 \mathrm{MeV}$ for the free proton. Adjusting Eq. 8 to this lower energy results in a $\sim 5 \%$ drop in the measured cross section to $90.1 \pm 8.6 \pm 3.6 \mathrm{nb} / \mathrm{sr}$. The ratio of the measured to effective free values is $1.35 \pm 0.18$, indicating they are not in good agreement. Model-dependence in extracting effective free-neutron values from quasi-free should be minimized by using the ratio of quasi-free cross sections, as discussed above. The dispersion calculations of L'vov et al. 18] predict a free value of $74.2 \mathrm{nb} / \mathrm{sr}$ for $(\bar{\alpha}-\bar{\beta})_{p}=10$.

Eqs. 7 and 10 can also be used to determine the effective free cross section for the neutron at $\theta_{\gamma^{\prime}}=-135^{\circ}$ :

$$
\frac{d \sigma\left(\gamma n \rightarrow \gamma^{\prime} n\right)}{d \Omega_{\gamma}}=39.5 \pm 8.5 \pm 3.6 \mathrm{nb} / \mathrm{sr},
$$

corresponding to $\mathrm{E}_{\gamma}=244.6 \mathrm{MeV}$ for the free neutron. This is the first experimental determination of the cross section for Compton scattering from the free neutron. An alternate method for extracting the free-neutron cross section from the measured values is to take the ratio of free to quasi-free cross sections for the proton (Eqs. B and 9) and multiply by the quasi-free neutron cross section (Eq. 10), resulting in $55.8 \pm 13.5 \pm 4.9$. The ratio of the extracted free values $1.41 \pm 0.47$ is consistent with unity, albeit with large errors. The dispersion calculations of L'vov et al. [18] predict a free value of $45.7 \mathrm{nb} / \mathrm{sr}$ (for $\left.(\bar{\alpha}-\bar{\beta})_{n}=10\right)$ in agreement with the experimental results.

In summary, the polarizabilities of the bound proton and neutron have, for the first time, been simultaneously extracted from quasi-free Compton scattering measurements. The values for the neutron (Eq. 13) are consistent with those known for the free proton (Eq. 5), as expected from charge-symmetry arguments, and are in accord with recent ChPT predictions.

The authors would like to thank M.I. Levchuk, A.I. L'vov, and V.A. Petrun'kin for supplying the results from their theoretical calculations. N.R.K. would like to thank M.I.L. for many useful discussions.

This work was supported in part by grants from the Natural Science and Engineering Research Council of Canada and the National Science Foundation (USA).

[1] B.E. MacGibbon et al., Phys. Rev. C 52, 2097 (1995).

[2] V.A. Petrun'kin, Sov. J. Part. Nucl. 11, 278 (1981).

[3] D. Babusci, G. Giordano, and G. Matone, Phys. Rev. C 57, 291 (1998).

[4] M.I. Levchuk and A.I. L'vov, Nucl. Phys. A (in press).

[5] F. Wissmann, M.I. Levchuk, M. Schumacher, Eur. Phys. J. A 1, 193 (1998).

[6] J. Schmiedmayer, P. Reihs, J.A. Harvey, and N.W. Hill, Phys. Rev. Lett. 66, 1015 (1991).

[7] L. Koester et al., Phys. Rev. C 51, 3363 (1995).

[8] T.L. Enik, L.V. Mitsyna, V.G. Nikolenko, A.B. Popov, and G.S. Samosvat, Sov. J. Nucl. Phys. 60, 567 (1997).

[9] M.A. Lucas, Ph.D. Thesis, University of Illinois, 1994.

[10] D.L. Hornidge et al., Phys. Rev. Lett. 84, 2334 (2000).

[11] K.W. Rose et al., Nucl. Phys. A514, 621 (1990).

[12] M.I. Levchuk, A.I. L'vov, and V.A. Petrun'kin, FewBody Sys. 16, 101 (1994).

[13] F. Wissmann et al., Nucl. Phys. 660, 232 (1999).

[14] E. Korkmaz et al., Nucl. Instrum. Methods Phys. Res. Sect. A 431, 446 (1999).

[15] J.P. Miller et al., Nucl. Instrum. Methods Phys. Res. Sect. A 270, 431 (1988).

[16] E.L. Hallin et al.,, Phys. Rev. C 48, 1497 (1993).

[17] A.M. Sandorfi, J. Tonnison, S. Hoblit, Few Body Sys., Suppl. 11, 144 (1999); S. Hoblit, private communication.

[18] A.I. L'vov, V.A. Petrun'kin, M. Schumacher, Phys. Rev. C 55, 359 (1997).

[19] J. Tonnison, A.M. Sandorfi, S. Hoblit, A.M. Nathan, Phys. Rev. Lett. 80, 4382 (1998).

[20] V. Bernard, N. Kaiser, U.-G. Mei $\beta$ ner, Int. J. Mod. Phys. E4, 193 (1995). 